\title{
Two-Dimensional FDTD Model of Antipodal ELF Propagation and Schumann Resonance of the Earth
}

\author{
Jamesina J. Simpson and Allen Taflove, Fellow, IEEE
}

\begin{abstract}
This letter reports the initial application of the finitedifference time-domain (FDTD) method to model extremely lowfrequency (ELF) propagation around the entire Earth. Periodic boundary conditions are used in conjunction with a variable-cell two-dimensional TM FDTD grid, which wraps around the complete Earth sphere. The model is verified by numerical studies of antipodal propagation and the Schumann resonance. This model may be significant because it points the way toward direct threedimensional FDTD calculation of round-the-world ELF propagation, accounting for arbitrary horizontal as well as vertical geometrical and electrical inhomogeneities of the ionosphere, continents, and oceans.
\end{abstract}

Index Terms-Antipodal propagation, Earth, ELF, finite difference time domain (FDTD), Schumann resonance.

\section{INTRODUCTION}

$\mathbf{P}$ ROPAGATION models of extremely low-frequency (ELF: $3 \mathrm{~Hz}-3 \mathrm{kHz}$ ) and very low-frequency (VLF: 3-30 kHz) electromagnetic waves in the Earth-ionosphere waveguide are primarily based upon frequency-domain waveguide mode theory [1], [2]. Recently, however, Cummer [3] reported applying the finite-difference time-domain (FDTD) method [4]. He used a two-dimensional (2-D) cylindrical-coordinate FDTD grid to investigate propagation over a $1000-\mathrm{km}$ ground path due to a vertical current channel simulating lightning discharge. Cummer found "extremely good" agreement between numerical mode theory and FDTD for ELF-VLF spectra below $10 \mathrm{kHz}$. Because FDTD permits straightforward modeling (with no increase in simulation time) of arbitrary horizontal as well as vertical inhomogeneities of the atmosphere and Earth, he concluded that "the simplicity of FDTD propagation modeling and ever-increasing computer power will probably make FDTD the technique of the future."

This letter reports a second step in the direction pointed out by Cummer. Namely, we describe the initial application of FDTD to model ELF propagation around the entire Earth. Periodic boundary conditions are used in conjunction with a variable-cell 2-D TM FDTD grid which wraps around the complete Earth sphere. The model is verified by numerical studies of antipodal propagation and the Schumann resonance.

\section{FDTD GRID}

To simplify computations, we seek to map the complete spherical surface of the Earth onto a logically Cartesian

Manuscript received January 28, 2002; revised April 5, 2002.

The authors are with the McCormick School of Engineering, Department of Electrical and Computer Engineering, Northwestern University, Evanston, IL 60208-3118 USA (e-mail: j-simpson@ northwestern.edu).

Digital Object Identifier 10.1109/LAWP.2002.805123
2-D, $2 m \times m$-cell TM FDTD grid having regular grid-cell coordinates, where $m$ is a power of 2 . Fig. 1 illustrates the general layout of the grid. The grid-cell position index in the west-to-east direction is $1 \leq i \leq 2 m$, and the grid-cell position index in the south-to-north direction is $1 \leq j \leq m$. We see that the grid cells follow along lines of constant latitude $\theta=$ constant, where $\theta$ is the usual spherical angle measured from the north pole; and along lines of constant longitude $\phi=$ constant, where $\phi$ is the usual spherical azimuthal angle measured from a specified prime meridian. In this manner, the grid shown in Fig. 1 is comprised of planar isosceles trapezoidal cells away from the north and south poles [Fig. 2(a)], and planar isosceles triangular cells at the poles [Fig. 2(b)].

We choose to have the same angular increment in latitude $\Delta \theta=\pi / m$ for each cell in the grid. Thus, the south-north span of each trapezoidal or triangular grid cell is $\Delta_{\mathrm{s}-\mathrm{n}}=\pi R_{\mathrm{E}} / m$, where $R_{\mathrm{E}}$ is the Earth's radius. To maintain square or nearly square grid cells near the equator, we select the baseline value of the angular increment in longitude $\Delta \phi$ to equal $\Delta \theta$. However, this causes the west-east span of each cell, $\Delta_{\mathrm{w}-\mathrm{e}}=R_{\mathrm{E}} \Delta \phi \sin \theta$, to be a function of $\theta$. This could be troublesome for cells near the north and south poles where $\theta \rightarrow 0$ and $\theta \rightarrow \pi$, respectively. There, the geometrical eccentricity of each cell, $\Delta_{\mathrm{s}-\mathrm{n}} / \Delta_{\mathrm{w}-\mathrm{e}}=\Delta \theta /(\Delta \phi \sin \theta)$, would become quite large and the numerical stability and efficiency of the FDTD algorithm would be degraded. An algorithmic means to deal with this problem will be discussed below.

The wrap-around or joining of the grid is along a specific line of constant longitude, or meridian. As discussed below, this joining is, in effect, a periodic boundary condition applied at each $j$ row of grid cells, whether trapezoids or triangles.

\section{FDTD ALGORITHM}

\section{A. Basic Algorithm}

Given the above assumptions, Ampere's Law in integral form [4] can be applied to develop an FDTD time-stepping relation for the electric field $E_{z}$ at the center of the $(i, j)$ th trapezoidal grid cell. For example, referring to Fig. 2(a), we have

$$
\begin{aligned}
& E_{z}^{n+1}(i, j)=E_{z}^{n}(i, j)+\frac{\Delta t}{\varepsilon_{0} S(j)} \\
& \cdot\left\{\begin{array}{l}
H_{x}^{n+0.5}(i, j-0.5) \Delta_{\mathrm{w}-\mathrm{e}}(j-0.5)- \\
H_{x}^{n+0.5}(i, j+0.5) \Delta_{\mathrm{w}-\mathrm{e}}(j+0.5)+ \\
{\left[H_{y}^{n+0.5}(i+0.5, j)-H_{y}^{n+0.5}(i-0.5, j)\right] \Delta_{\mathrm{s}-\mathrm{n}}}
\end{array}\right\}
\end{aligned}
$$




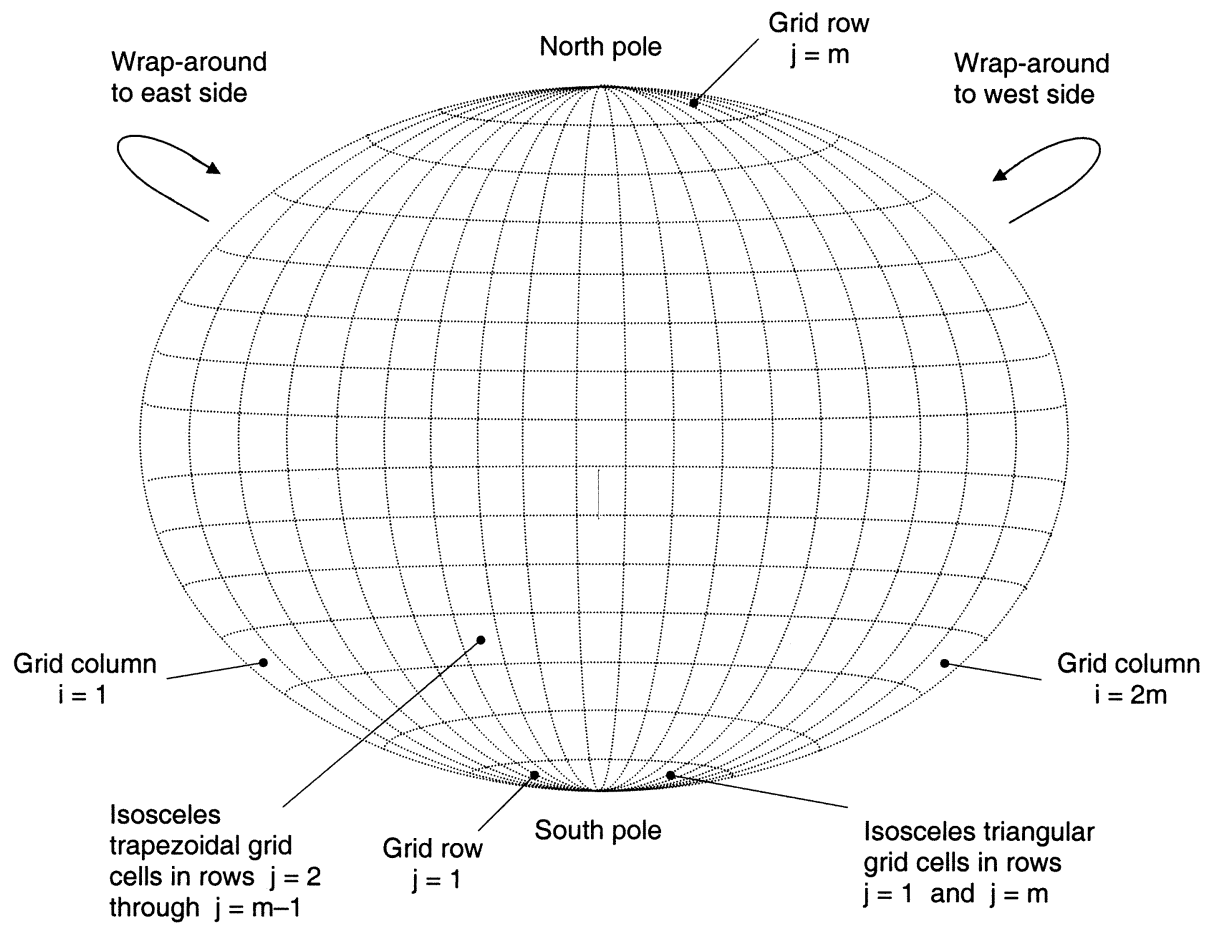

Fig. 1. General layout of the of the 2-D FDTD grid to cover the complete Earth sphere. Each grid cell is planar.

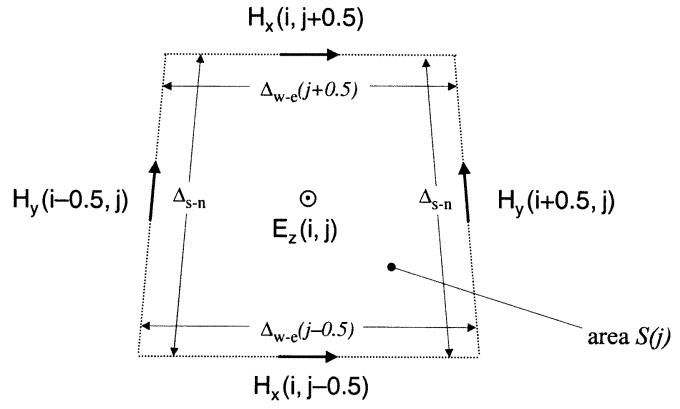

(a)

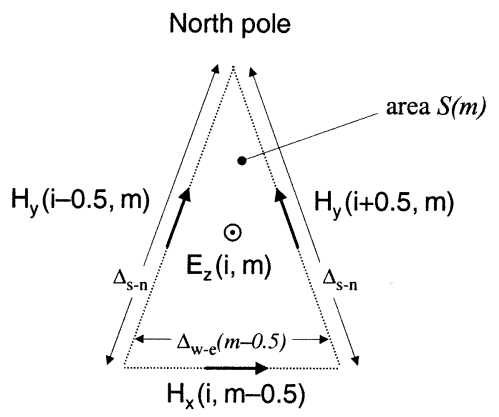

(b)

Fig. 2. Details of the grid-cell geometry. (a) Isosceles trapezoidal cell in the northern hemisphere away from the north pole. (b) Isosceles triangular cell at the north pole.

where $\Delta t$ is the time step and

$$
\begin{aligned}
\Delta_{\mathrm{w}-\mathrm{e}}(j+0.5)= & R_{\mathrm{E}} \Delta \phi \sin [(m-j) \pi / m] \\
\Delta_{\mathrm{w}-\mathrm{e}}(j-0.5)= & R_{\mathrm{E}} \Delta \phi \sin [(m-j+1) \pi / m] \\
S(j) \cong & {\left[\Delta_{\mathrm{w}-\mathrm{e}}(j-0.5)+\Delta_{\mathrm{w}-\mathrm{e}}(j+0.5)\right] } \\
& \cdot \Delta_{\mathrm{s}-\mathrm{n}} / 2 .
\end{aligned}
$$

Similarly, referring to Fig. 2(b), the update for $E_{z}$ at the center of the $i$ th triangular grid cell at the north pole $(j=m)$ is

$$
\begin{aligned}
& E_{z}^{n+1}(i, m)=E_{z}^{n}(i, m)+\frac{\Delta t}{\varepsilon_{0} S(m)} \\
& \cdot\left\{\begin{array}{l}
H_{x}^{n+0.5}(i, m-0.5) \Delta_{\mathrm{w}-\mathrm{e}}(m-0.5)+ \\
{\left[H_{y}^{n+0.5}(i+0.5, m)-H_{y}^{n+0.5}(i-0.5, m)\right] \Delta_{\mathrm{s}-\mathrm{n}}}
\end{array}\right\}
\end{aligned}
$$

where $\Delta_{\mathrm{w}-\mathrm{e}}(m-0.5)$ is given by (2b) for the case $j=m$ and

$$
\begin{aligned}
& S(m)=\frac{\Delta_{\mathrm{w}-\mathrm{e}}(m-0.5) \Delta_{\mathrm{s}-\mathrm{n}}}{2} \\
& \cdot \sin \left\{\cos ^{-1}\left[\frac{\Delta_{\mathrm{w}-\mathrm{e}}(m-0.5)}{2 \Delta_{\mathrm{s}-\mathrm{n}}}\right]\right\} .
\end{aligned}
$$

Expressions analogous to (3) and (4) can be derived for the $i$ th triangular grid cell at the south pole $(j=1)$.

The basic FDTD time-stepping algorithm is completed by specifying the updates for the $H_{x}$ and $H_{y}$ fields. For example, referring to the trapezoidal grid cell shown in Fig. 2(a), we have

$$
\begin{aligned}
H_{x}^{n+1.5}(i, j-0.5)= & H_{x}^{n+0.5}(i, j-0.5)+\frac{\Delta t}{\mu_{0} \Delta_{\mathrm{s}-\mathrm{n}}} \\
& \cdot\left[E_{z}^{n+1}(i, j-1)-E_{z}^{n+1}(i, j)\right] \\
H_{y}^{n+1.5}(i+0.5, j)= & H_{y}^{n+0.5}(i+0.5, j)+\frac{\Delta t}{\mu_{0} \Delta_{\mathrm{w}-\mathrm{e}}(j)} \\
& \cdot\left[E_{z}^{n+1}(i+1, j)-E_{z}^{n+1}(i, j)\right] .
\end{aligned}
$$

$H_{x}$ and $H_{y}$ updates for a triangular grid cell at the north pole shown in Fig. 2(b) are given by (5) and (6) with $j=m$ and at the south pole with $j=1$. 


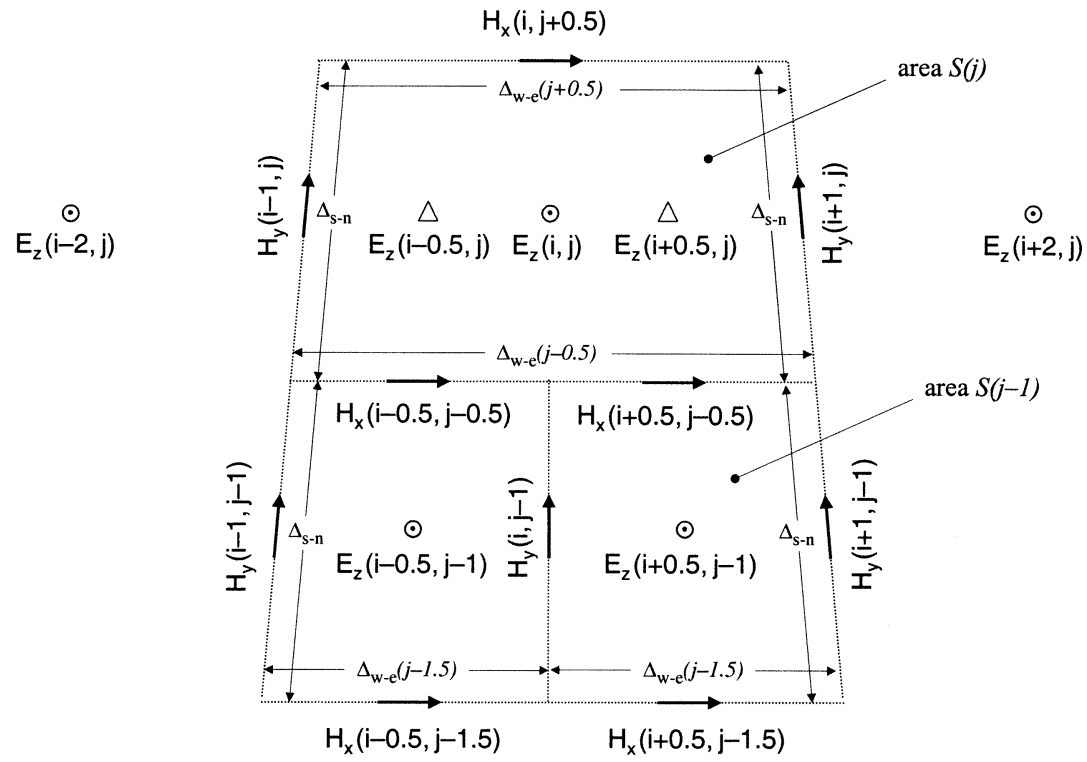

Fig. 3. Details of the grid-cell geometry in the northern hemisphere at the transition between two adjacent regular cells and a single cell spanning twice the distance in the east-west direction.

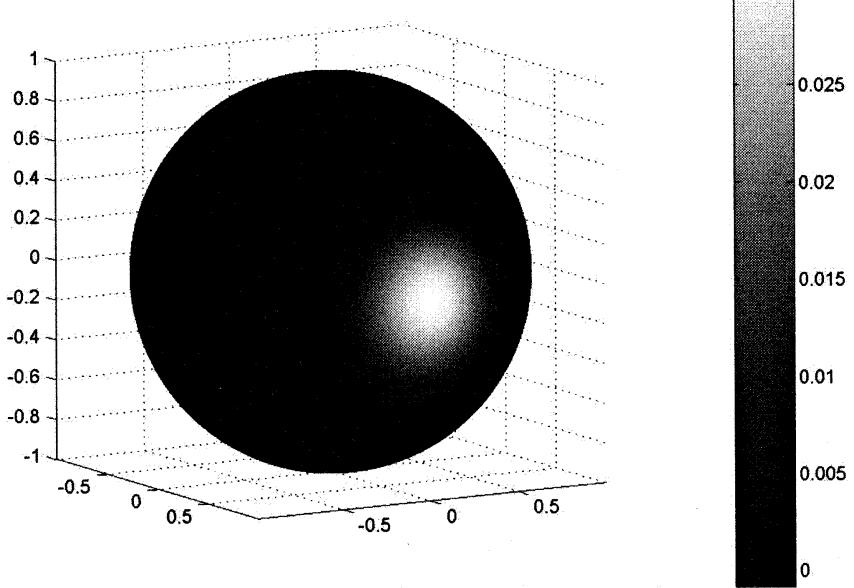

Fig. 4. Grayscale visualization of the electric field projected onto the surface of the Earth sphere at the time of its initial peak value at the antipode.

\section{B. Merging Trapezoidal Grid Cells Approaching the Poles}

As stated earlier, near the north and south poles, the geometrical eccentricity of each trapezoidal cell becomes quite large for a constant value of $\Delta \phi$. Fig. 3 illustrates a means to mitigate this problem by merging pairs of adjacent cells in the west-east direction. This process can be repeated several times as the grid approaches a pole.

The required algorithm is now presented. Applying Ampere's Law in integral form, the time-stepping relation for the $E_{z}$ field at the center of the merged cell is

$$
\begin{aligned}
E_{z}^{n+1} & (i, j)=E_{z}^{n}(i, j)+\frac{\Delta t}{\varepsilon_{0} S(j)} \\
& \cdot\left\{\begin{array}{l}
H_{x}^{n+0.5}(i-0.5, j-0.5) \Delta_{\mathrm{w}-\mathrm{e}}(j-0.5) / 2+ \\
H_{x}^{n+0.5}(i+0.5, j-0.5) \Delta_{\mathrm{w}-\mathrm{e}}(j-0.5) / 2- \\
H_{x}^{n+0.5}(i, j+0.5) \Delta_{\mathrm{w}-\mathrm{e}}(j+0.5)+ \\
{\left[H_{y}^{n+0.5}(i+1, j)-H_{y}^{n+0.5}(i-1, j)\right] \Delta_{\mathrm{s}-\mathrm{n}}}
\end{array}\right\} .
\end{aligned}
$$

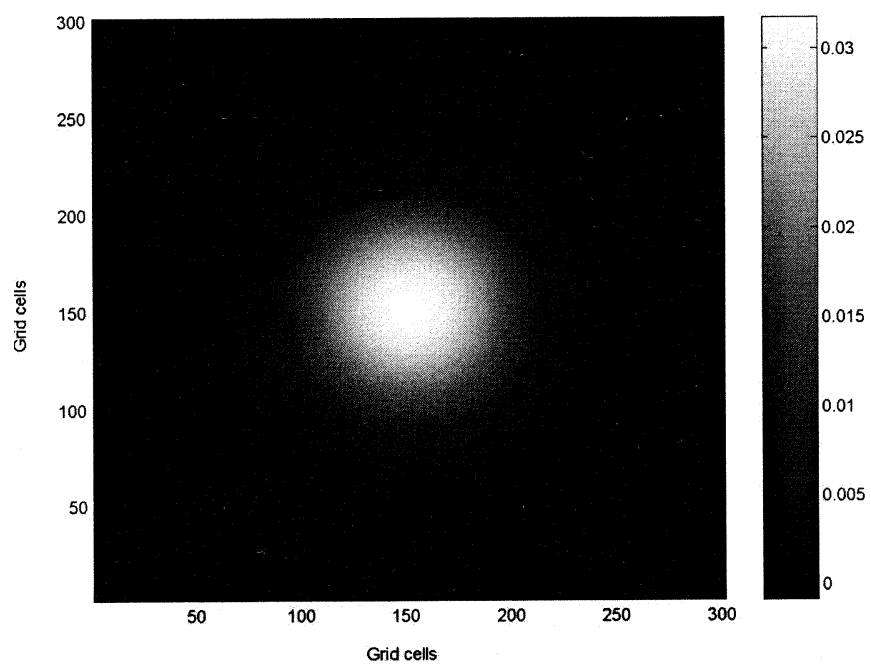

Fig. 5. Grayscale visualization of the electric field in the region immediately surrounding the antipode at the time of its initial peak value.

$E_{z}$ fields within the merged cell required for the subsequent magnetic-field updates are obtained by linearly interpolating the $E_{z}$ field quantities calculated in (7), for example

$$
\begin{aligned}
& E_{z}^{n+1}(i-0.5, j) \\
& \quad=\left[3 E_{z}^{n+1}(i, j)+E_{z}^{n+1}(i-2, j)\right] / 4
\end{aligned}
$$

Now, the time-stepping algorithm for the merged cell can be completed by specifying the updates for the $H_{x}$ and $H_{y}$ fields at the periphery of the cell, for example

$$
\begin{aligned}
H_{x}^{n+1.5}(i-0.5, j-0.5) \\
=H_{x}^{n+0.5}(i-0.5, j-0.5)+\frac{\Delta t}{\mu_{0} \Delta_{\mathrm{s}-\mathrm{n}}} \\
\quad \cdot\left[E_{z}^{n+1}(i-0.5, j-1)-E_{z}^{n+1}(i-0.5, j)\right]
\end{aligned}
$$




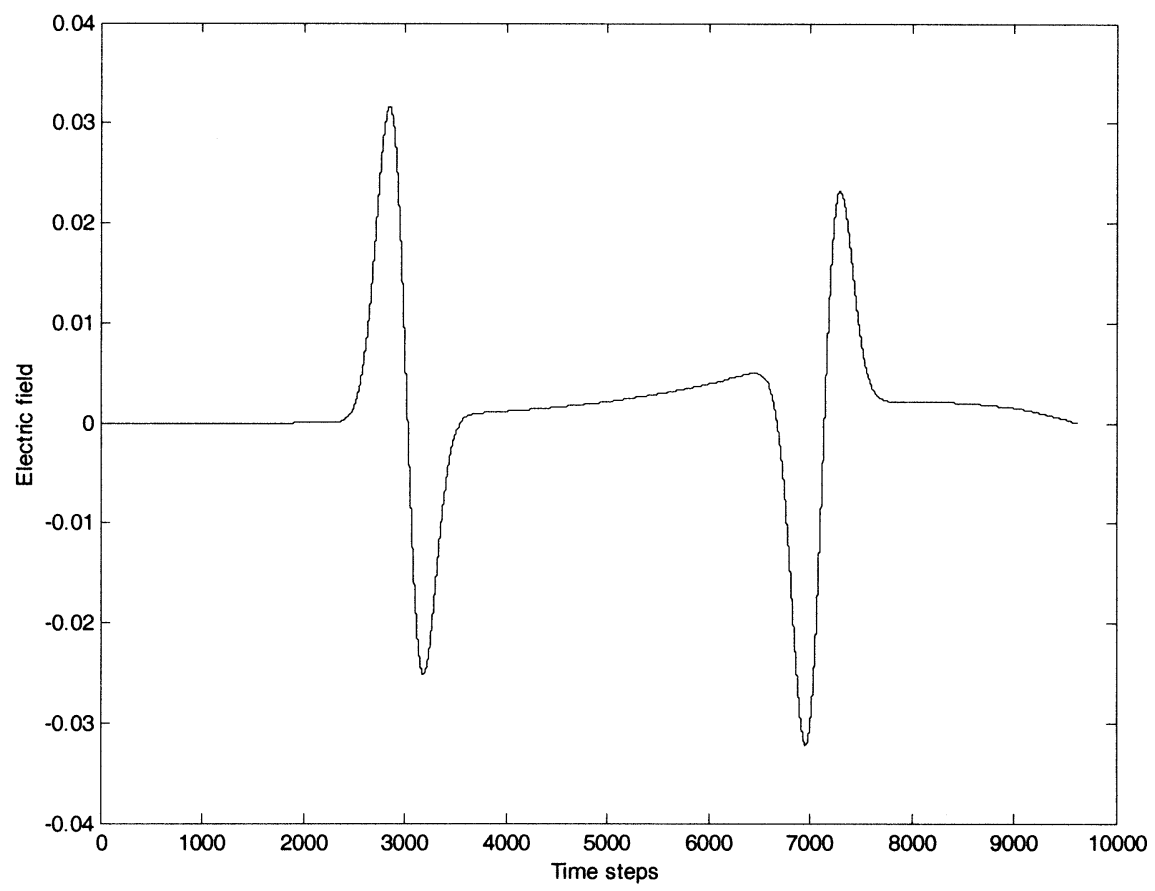

Fig. 6. Calculated time waveform of the electric field at the antipode for the first two circumnavigations of the Earth sphere.

$$
\begin{aligned}
H_{y}^{n+1.5}(i+1, j) \\
=H_{y}^{n+0.5}(i+1, j)+\frac{\Delta t}{\mu_{0} \Delta_{\mathrm{w}-\mathrm{e}}(j)} \\
\cdot\left[E_{z}^{n+1}(i+2, j)-E_{z}^{n+1}(i, j)\right] .
\end{aligned}
$$

\section{West-East Grid Wrap Around}

The grid wrap around (periodic boundary condition) in the west-east direction completes the FDTD algorithm. For each row $j$ in Fig. 1, this is implemented by

$$
\begin{gathered}
H_{y}^{n+1.5}(0.5, j)=H_{y}^{n+0.5}(0.5, j)+\frac{\Delta t}{\mu_{0} \Delta_{\mathrm{w}-\mathrm{e}}(j)} \\
\cdot\left[E_{z}^{n+1}(1, j)-E_{z}^{n+1}(2 m, j)\right] . \\
H_{y}^{n+1.5}(2 m+0.5, j)=H_{y}^{n+1.5}(0.5, j) .
\end{gathered}
$$

\section{RESULTS}

We first report the results of a numerical experiment designed to test the accuracy of our FDTD numerical model for an impulsive circular cylindrical wave. The idea here is to track the wave as it propagates radially outward from a filamentary current source, travels around the Earth-sphere model, and then propagates radially inward to the antipode. Results are shown for a lossless $1024 \times 512$ grid spanning the Earth sphere.

Figs. 4 and 5 are grayscale visualizations of the electric field at the time of its initial peak value at the antipode. The high de- gree of azimuthal symmetry is a measure of the isotropy of the numerical wave propagation within the model. Note that this propagation occurs concurrently through both uniform grid regions near the equator and complicated polar regions having a nonuniform mesh of cells with variable eccentricity, mergers, and transitions from trapezoids to triangles.

Fig. 6 graphs the calculated time waveform of the electric field at the antipode for the first two circumnavigations of the Earth sphere. This figure shows the $180^{\circ}$ phase reversals of the electric field at the antipode first calculated by Wait [5]. Further, this figure shows a 0.134 -s circumnavigation period corresponding to a fundamental Earth resonance of $7.46 \mathrm{~Hz}$.

\section{ONGOING WORK}

We are extending the 2-D FDTD grid discussed here to a fully 3-D space lattice filling the complete Earth-ionosphere volume. This will permit accounting of vertical as well as horizontal inhomogeneities of the excitation, atmosphere, and Earth. We expect that this model could eventually be coupled with emerging whole-Earth geophysical codes [6].

\section{REFERENCES}

[1] K. G. Budden, "The influence of the Earth's magnetic field on radio propagation by wave-guide modes," Proc. Roy. Soc. A, vol. 265, p. 538, 1962.

[2] J. R. Wait, Electromagnetic Waves in Stratified Media. New York: Pergamon, 1970.

[3] S. A. Cummer, "Modeling electromagnetic propagation in the Earthionosphere waveguide," IEEE Trans. Antennas Propagat., vol. 48, p. 1420, Sept. 2000.

[4] A. Taflove and S. C. Hagness, Computational Electrodynamics: The Finite-Difference Time-Domain Method, 2nd ed. Norwood, MA: Artech House, 2000.

[5] J. R. Wait, "Distortion of ELF pulses after propagation through an antipode," J. Geophys. Res., vol. 74, pp. 2982-2986, 1969.

[6] APEC cooperation for earthquake simulation (ACES). World Wide Web URL. [Online]. Available: http://shake2.earthsciences.uq.edu.au/ACES/ 